\title{
Transcriptional and translational mechanisms contribute to regulate the expression of Discs Large 1 protein during different biological processes
}

\begin{abstract}
Human discslarge(DLG1) has been demonstrated to be involved in cell polarity and maintenance of tissue architecture. However, the mechanisms controlling DLG1 expression are not fully understood. This is relevant as DLG1 is lost during the later stages of malignant progression. We initiated a series of studies to analyse the mechanisms regulating DLG1 expression. We have previously reported the identification of an alternative splicing event in the $5^{\prime}$ untranslated region (5'-UTR) of DLG1 mRNA that generates transcripts with two different $5^{\prime}$-UTR (short and large $5^{\prime}$-UTR variants). In this study, we further examined the impact of the DLG1 transcription and the role of the differential expression of the alternative $5^{\prime}$-UTRs on DLG1 protein levels. We analysed these mechanisms during cell processes like differentiation, cell cycle progression and cell-cell contact formation, where the importance of DLG1 activities was previously established. The data presented in this report suggest that the transcriptional regulation of DLG1 strongly contributes to DLG1 abundance and that differential expression of alternative 5 '-UTRs with different translational properties, also cooperates, depending on the cell type and cell situation. This study provides new evidence for understanding the transcriptional regulation of DLG1 and the changes in DLG1 expression during different biological processes.
\end{abstract}

Keywords: 5'-UTR; DLG1; expression; polarity; transcription regulation.

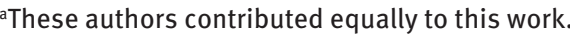

*Corresponding author: Daniela Gardiol, Instituto de Biología Molecular y Celular de Rosario-CONICET, Facultad de Ciencias Bioquímicas y Farmacéuticas, Universidad Nacional de Rosario, Suipacha 590, 2000 Rosario, Argentina,

e-mail: gardiol@ibr-conicet.gov.ar

Federico Marziali, Ana Laura Cavatorta, Marina Bugnon Valdano and Florencia Facciuto: Instituto de Biología Molecular y Celular de Rosario-CONICET, Facultad de Ciencias Bioquímicas y Farmacéuticas, Universidad Nacional de Rosario, Suipacha 590, 2000 Rosario, Argentina
DOI 10.1515/hsz-2014-0286

Received December 1, 2014; accepted February 2, 2015

\section{Introduction}

The human discs large protein (DLG1) is an ortholog of DLG protein from Drosophila (DLG-A) and belongs to the membrane-associated guanylate kinase family of proteins whose members are characterised by the presence of different protein interaction modules (for a review see Roberts et al., 2012). These interaction modules, including the PSD-95/DLG/ZO-1 (PDZ) domains, allow the formation of multiprotein complexes at specialised sites of the cell, which are involved in functional signal transduction networks. In Drosophila, DLG was demonstrated to be a key regulator of both cell proliferation and polarity and loss of DLG functions was associated with neoplastic transformation (Bilder, 2004). In mammalian epithelial cells, DLG1 localises in the cytoplasm and at sites of cell contacts, in association with adherens junctions (Laprise et al., 2004) and, together with Scribble and lethal giant larvae (Lgl), is a component of the Scribble polarity complex, which is crucial for the establishment and maintenance of apicobasal polarity (Assemat et al., 2008). Since DLG1 was first detected and cloned, the mRNA was shown to be alternatively spliced, leading to several DLG1 isoforms that display different cell localisation and functions (Lue et al., 1994; McLaughlin et al., 2002). Moreover, DLG1 protein was shown to be ubiquitously expressed and to play an important role in regulating different activities in a variety of cells, including epithelial cells, lymphocytes and neurons.

DLG1 interacts with several proteins including the phosphatase and tensin homolog (PTEN) and Adenomatous polyposis coli (APC) oncosupressors, that participate of the control of cell proliferation (Sotelo et al., 2012). Moreover, these interactions were shown to be important 
for the negative regulation of cell growth (Ishidate et al., 2000; Valiente et al., 2005; Sotelo et al., 2012). However, even though most of the observations suggest oncosuppression functions for the mammalian DLG ortholog, as it was defined in Drosophila, there is evidence that it can behave like an oncoprotein according to the cellular context as, for example, in the presence of transforming viral proteins (Frese et al., 2006; Subbaiah et al., 2012).

Several reports using human biopsies have described changes for DLG1 expression during malignant progression (Facciuto et al., 2012). While a marked reduction of DLG1 levels in later stages and in poorly differentiated tumours was described, a high overexpression and mislocalisation of DLG1 at earlier stages of cervical, colon, breast and gastric cancers was observed (Watson et al., 2002; Boussioutas et al., 2003; Cavatorta et al., 2004; Fuja et al., 2004; Gardiol et al., 2006). However, the contribution of this high DLG1 expression to tumour prognosis, the mechanisms involved in the reduction of DLG1 levels at more severe stages and its precise relevance, remain unknown.

Even though great progress has been achieved for the understanding of the post-translational modifications involved in the regulation of DLG1 cell localisation and functions (Roberts et al., 2012), very little is known about the transcription patterns of DLG1 and its contribution to the control of DLG1 protein abundance in different situations. Few reports have demonstrated a down-regulation in DLG1 mRNA levels associated to transformation or in cells derived from some cancers (Mantovani et al., 2001; Vieira et al., 2008; Szymanowska-Narloch et al., 2013). However, an integrated picture of the mechanism controlling its expression in different biological processes is not available, even though it would be extremely relevant, according to the changes in DLG1 levels observed during malignant progression (Facciuto et al., 2012).

Accordingly, we initiated a series of studies to analyse the mechanism regulating DLG1 transcription. We reported previously the cloning and functional characterisation of the promoter region of DGL1 and demonstrated its regulation by the Snai1 transcription factor, which is involved in the epithelial mesenchymal transition (Cavatorta et al., 2008). Moreover, we identified the presence of an alternative splicing event in the $5^{\prime}$-UTR of DLG1 that generates two different transcripts: the short and large variants (Figure 1A). The exclusion of the alternative spliced exon in the short form increases the efficiency of DLG 1 translation, indicating the participation of the $5^{\prime}$-UTR in the control of DLG1 abundance (Cavatorta et al., 2011). We showed that this event is significant in the regulation of DLG1 levels in transformed cells, in agreement with the concept that many oncogenes and tumour suppressor genes express complex $5^{\prime}$-UTRs that participate during carcinogenesis. In fact, translation initiation is highly sensitive to features found in the $5^{\prime}$-UTR, like the presence of AUG(s) upstream of the main ORF, as identified in the large version of the 5'-UTR DLG1 (Cavatorta et al., 2011; Dikstein, 2012). The splicing identified does
A

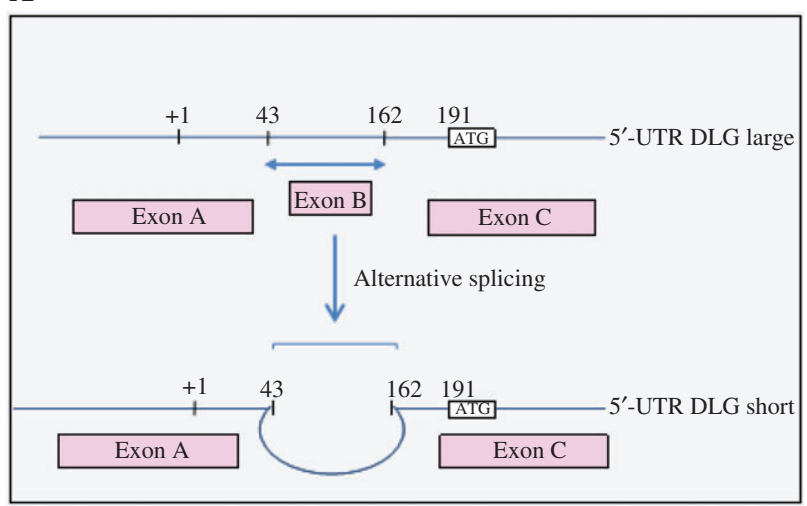

B

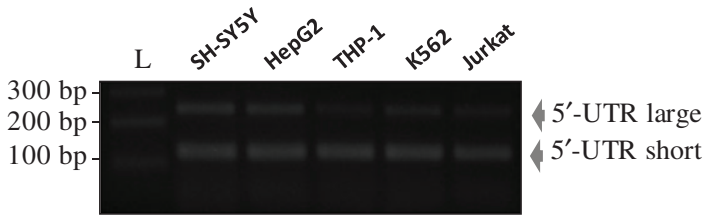

Figure 1: The alternative splicing in the DLG1 5'-UTR is conserved among different cell lines.

(A) Schematic representation of $5^{\prime}$-UTR region of DLG1 and mRNA splice variants. The reported transcription start site is marked as +1 (GeneBank ID: U13896 and U13897). Exons containing the $5^{\prime}$-UTR region are indicated. The DLG 5'-UTR Large includes Exon A, Exon B and part of Exon C (Cavatorta et al., 2011). In the short version of $5^{\prime}$-UTR DLG the Exon B is absent. The length of each exon is not drawn to scale. (B) Total RNA of the indicated cell lines was extracted and subjected to RT-PCR as described (Cavatorta et al., 2011). Both large and short 5'UTR forms of DLG1 are shown as upper and lower bands, respectively. The cells used were: HepG2 cells (human hepatocellular carcinoma); SH-SY5Y cells (human neuroblastoma); Jurkat cells (human T cell leukemia); K562 cells (human myelogenous leukemia) and THP-1 cells (human monocyte-derived leukemia). L, DNA ladder. 
not involve the coding region and though, the transcripts containing any of the $5^{\prime}$-UTR versions should codify for the same protein, although with different translation efficiency. However, the complete functional roles of the $5^{\prime}$ alternative transcripts of DLG1 were not fully elucidated nor their significance in different cell types.

In this study, we further investigated the impact of the transcription and the differential expression of the alternative $5^{\prime}$-UTRs on DLG1 protein levels. We analysed these parameters during cell processes like differentiation, cell cycle progression and adherence, where the importance of DLG1 activities and subcellular distribution was previously established. We demonstrated that the transcriptional regulation of DLG1 strongly contributes to DLG1 abundance and the alternative splicing event in the $5^{\prime}$-UTR also cooperates, depending on the cell context. This report offers new evidence that contributes to the understanding of the pattern of DLG1 expression levels.

\section{Results}

\section{The alternative DLG1 5'-UTRs are expressed in cell lines from different tissues}

Our previous analyses of DLG1 expression were mainly focused on epithelial cells (Cavatorta et al., 2011). However, this protein was shown to be expressed in several tissues (Lue et al., 1994) and many reports indicate that DLG1 is also involved in many activities in cells such as lymphocytes and neurons. Therefore, we wanted to assess whether the alternative short and large $5^{\prime}$-UTR formerly identified (Figure 1A) were also differentially expressed in cancer cells derived from diverse tissues, where the importance of DLG1 activities was previously established. We performed RT-PCR assays for the differential amplification of both DLG1 5'-UTRs using RNA from A375, HepG2, Jurkat, SH-SY5Y, THP-1 and K562 cell lines. We included a reverse common primer and isoform-specific forward primers for each UTR, as described previously (Cavatorta et al., 2011). As can be seen in Figure 1B, both large and short $5^{\prime}$-UTR forms of DLG1 transcripts, shown as upper and lower major bands respectively, could be detected in all cell lines analysed, the short version being the most abundant variant in every case. This result indicates that the alternative splicing in the DLG1 $5^{\prime}$-UTR is conserved among the different cancer cell lines analysed, with potential consequences on the regulation of DLG1 levels.

\section{Transcription and differential expression of alternative 5'-UTRs regulate DLG1 protein levels at high cell confluence}

The DLG1 oncosuppressor represents a key component in the formation of adherens junctions of epithelial cells, whose levels increase with cell confluence, in agreement with the requirement of a greater number of cell junctions (Mantovani et al., 2001). In fact, DLG1 protein was shown to be stabilised upon increased cell contacts, suggesting the existence of cellular mechanisms regulating DLG1 abundance in response to cellcell contacts. To elucidate whether the transcription level and the differential expression of alternative 5'-UTRs may contribute to that observation, we monitored the amount of total DLG1 mRNA and of each DLG1 $5^{\prime}$-UTR isoform, in cells growing at low and high densities. Different amounts of Caco-2 cells were plated and harvested for protein and RNA analysis after $24 \mathrm{~h}$, at approximately 25\% (low confluence, L) and 80\% (high confluence, $\mathrm{H}$ ) confluence.

We then performed Western blotting analysis to investigate protein levels and RT-qPCR assays to assess the total DLG1 RNA level and, specifically, the expression of each DLG1 5'-UTR variant from cDNA isolated from cells cultured to the different densities. RT-qPCR analyses were performed using primers designed on DLG1 reading frame (present in all DLG1 messages) and specific primers for each 5'-UTR version. Succinate dehydrogenase (SDH) was used as reference gene (Cavatorta et al., 2011).

As can be appreciated from the results in Figure 2, DLG1 protein abundance is increased as cells become more confluent, as previously reported (Figure 2A) (Mantovani et al., 2001), together with a global increase of approximately three-fold in DLG1 transcription (Figure 2B). Interestingly, when assessing the amount of each 5'-UTR isoform, both variants were increased when cell confluence was raised, yet to a different extent for each one (Figure 2C). The short DLG1 5'-UTR, associated to a major translation efficiency, was up-regulated to a higher degree compared to the large $5^{\prime}$-UTR (Figure 2C). This is reflected in the increase of $5^{\prime}$-UTR short/large ratio at high cell confluence (Figure 2C). This result indicates that the short and large DLG1 5-UTRs are differentially expressed during the increment of the cell confluence and, in addition to gene transcription gain, the alternative 5 -UTR splicing occurs at a higher frequency, most likely contributing to regulate DLG1 protein levels. 
A
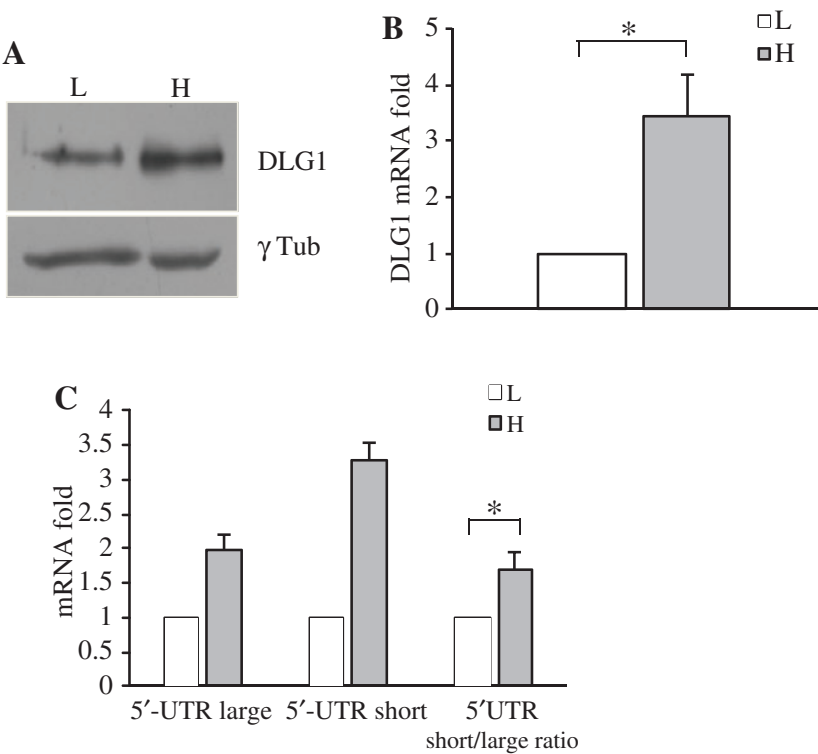

Figure 2: Transcription and differential expression of alternative $5^{\prime} U T R s$ regulate DLG1 protein levels at high cell confluence. Caco-2 cells were cultured at different densities and harvested for protein and RNA analysis at low confluence (L) or high confluence $(H)$. (A) DLG1 protein levels are increased at high cell density. Protein levels were ascertained by Western blotting analysis with anti-DLG1 (upper panel) or anti- $\gamma$ tubulin antibodies (as loading control, lower panel). (B) DLG1 transcription rises at high confluence. Changes in total DLG1 mRNA level were quantified by RT-qPCR as described in Materials and methods. DLG1 mRNA contents were normalised to the SDH mRNA for all samples and the relative DLG1 mRNA amount at $L$ was arbitrarily considered to be $1 .{ }^{\star} p<0.05$. (C) The alternative splicing in the DLG1 $5^{\prime}$-UTR is increased at high cell confluence. Variation of each $5^{\prime}$-UTR mRNA isoform level for $H$ relative to $L$ cell confluence (data arbitrarily considered to be 1) is shown. For quantification of each alternative DLG1 5'-UTR we performed RT-qPCR as described in Materials and methods. DLG1 $5^{\prime}$-UTR mRNA contents were normalised to the SDH mRNA contents for all samples. Relative short/large $5^{\prime}$-UTR mRNA fold is also shown for $L$ and $H$ cell confluence. Short/large DLG1 $5^{\prime}$-UTR mRNA at $L$ cell confluence was arbitrarily considered to be $1 .{ }^{*} p<0.05$. In all cases, results are shown as mean $\pm \mathrm{SE}$ and represent data from three independent experiments.

\section{Differential expression of DLG1 during cell cycle progression}

The increase in DLG1 levels as cells reached confluence may be related to a restriction in cell proliferation. Actually, previous studies have also shown that DLG1 can act to suppress G0/G1 to S phase transition linking DLG1 function with the control of cell cycle (Ishidate et al., 2000). Despite the relevance of this DLG1 activity, very little is known about the abundance of this protein during different phases of the cell cycle. To address this issue, we first synchronised Caco-2 cells by serum starvation, in order to obtain cell populations enriched in G0/G1 or $\mathrm{S}$ phases. We analysed the cell cycle profiles by flow cytometry and confirmed that $48 \mathrm{~h}$ after serum deprivation most of the cell population was in G0/G1 and $24 \mathrm{~h}$ after serum repletion the cells were highly enriched in $\mathrm{S}$ phases (see the Materials and methods section, Figure 3A). In addition, the expression of the S-associated protein, cyclin E, was used as a control of $\mathrm{S}$ phase induction (Figure 3B). Once these parameters were determined, we analysed DLG1 expression in both populations, harvesting cells at the time points described above. As shown in Figure 3B, when the majority of the cells are in the $\mathrm{S}$ phase, there is a clear decrease in DLG1 protein levels when compared to cells largely arrested in G0/G1. This result is in agreement with previous data indicating that DLG1 suppresses cell $S$ phase entry (Ishidate et al., 2000). We then employed RT-qPCR analysis to investigate the contribution of total DLG1 mRNA expression and as observed, the transcription level was significantly reduced in the $S$ phase population to 2.5-fold compared to the G0/G1 group (Figure 3C). Then, considering that the pool of transcripts would be constituted by the short and large 5'-UTR isoforms, we aimed to elucidate whether the relative proportion of these variants could change during the cell cycle progression, contributing to the changes in DLG1 protein abundance. We performed specific RT-qPCR for each isoform and the results showed that entry into $S$ phase entails a reduction in the levels of both transcripts at the same rate (Figure 3D), so no relevant changes in the short/large ratio were observed (Figure 3D). This would indicate that the frequency of the alternative splicing in the DLG1 5'-UTR would not be affected during the progression to the $S$ phase in the cell cycle, suggesting that the changes in DLG1 expression related to cell proliferation induction are more likely regulated at the transcriptional and not the post-transcriptional level.

In agreement with this, Zimmermann et al. have previously shown that the expression of Hugl-2 protein (human homolog to Lgl), a component of the same polarity complex that DLG1, is transcriptionally regulated in a negative manner by inductors of cell proliferation, like growth factors (Zimmermann et al., 2008). Therefore, in order to corroborate the data presented above we evaluated the effect of the addition of cell growth factors (CGF) on the expression of DLG1. Epithelial cells were cultured for $24 \mathrm{~h}$ in the presence of different quantities of the commercial Defined Keratinocyte-SFM growth factors mix, as indicated in Figure 4A. After 24 h, RNA extracts were prepared and the levels of DLG1 transcripts were ascertained for treated and untreated cells by RT-qPCR. As can 
A

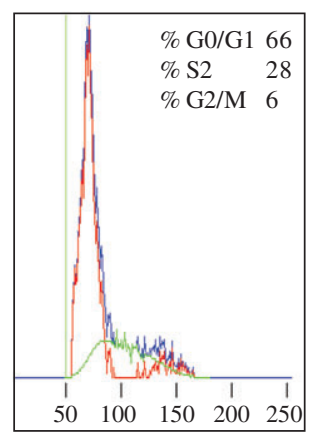

Serum deprivation

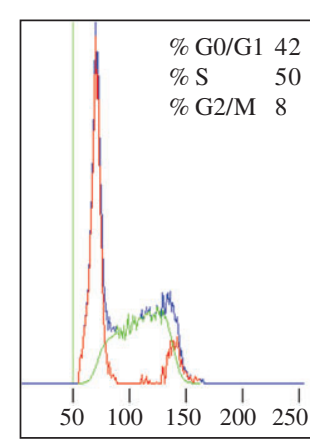

Serum repletion

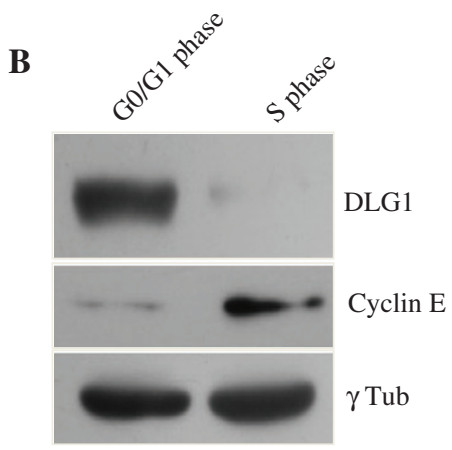

D

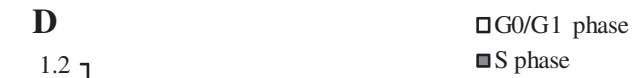

C

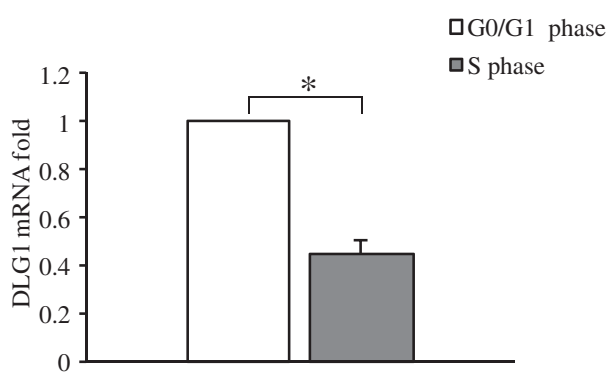

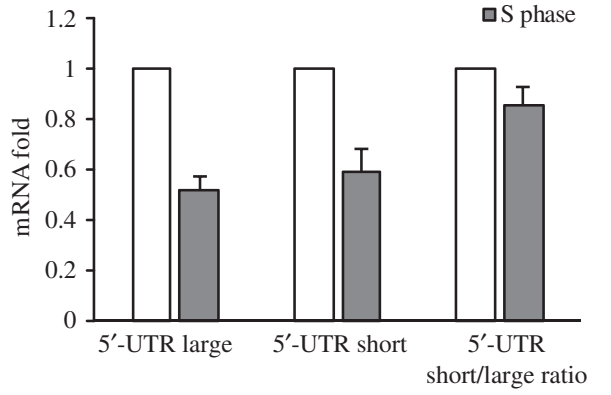

Figure 3: Changes in DLG1 expression during cell proliferation induction are more likely regulated at the transcriptional level.

(A) FACS analysis showing cell cycle progression. The cell cycle profiles of $\mathrm{CaCo}-2$ cells $48 \mathrm{~h}$ after serum cell deprivation (left panel) and $24 \mathrm{~h}$ after serum repletion (right panel) were analysed by flow cytometry as described in Materials and methods. The approximate percentages of cells in each of the major phases of the cell cycle are shown. The result is representative of three independent experiments. (B) DLG1 protein levels are decreased at the $\mathrm{S}$ phase of the cell cycle. Protein levels were ascertained by Western blotting analysis, using cells enriched in S or in G0/G1 phases of cell cycle, with anti-DLG1 (upper panel) or anti- $\gamma$ tubulin antibodies (as loading control, lower panel). The expression of the $\mathrm{S}$-associated protein, cyclin $\mathrm{E}$, was ascertained as a control of $\mathrm{S}$ phase induction (middle panel). (C) DLG1 transcription is reduced in cell S phase population. Changes in total DLG1 mRNA level were quantified by RT-qPCR as described in Materials and methods. DLG1 mRNA contents were normalised to the SDH mRNA for all samples and the relative DLG1 mRNA at G0/G1 phase was arbitrarily considered to be $1 .{ }^{\star} p<0.05$. (D) The alternative splicing in the DLG1 $5^{\prime}$-UTR is not affected during the induction of cell proliferation. Variation of each $5^{\prime}$-UTR mRNA isoform level for $\mathrm{S}$ phase relative to G0/G1 phase (data arbitrarily considered to be 1) is shown. For quantification of each alternative DLG1 $5^{\prime}$-UTR we performed RT-qPCR as described under Materials and methods. DLG1 $5^{\prime}$-UTR mRNA contents were normalised to the SDH mRNA contents for all samples. Relative short/large $5^{\prime}$-UTR mRNA fold is also shown for G0/G1 and S phases of cell cycle. Short/large DLG1 5'-UTR mRNA at G0/G1 phase was arbitrarily considered to be 1 . In all cases, results were shown as mean \pm SE and represent data from three independent experiments.

be appreciated in Figure 4A, the addition of CGF implies a significant reduction in endogenous DLG1 mRNA expression in a dose-dependent manner.

Next, we wanted to analyse the probable modulators participating in the down-regulation of DLG1 by the growth factors. We previously showed that Snai1 factor represses DLG1 transcription and, interestingly, other authors demonstrated that Snai1 levels increases by mitogen-induced cell proliferation (De Craene et al., 2005; Cavatorta et al., 2008; Zimmermann et al., 2008). Though, we performed RT-PCR to determine possible changes in Snai1 expression after treatment with the CGF. As shown in Figure 4B, the transcriptional expression of Snai1 was increased by the addition of the CGF and this also occurs in a dose-dependent manner.
Taken together, the overall results suggest that the induction of cell proliferation produce a decrease in DLG1 expression, and this down-regulation could probably be mediated by an increase in the levels of Snai1 transcription repressor.

\section{Phorbol myristate acetate (PMA)-induced differentiation of THP-1 cells results in reduction of DLG1 expression}

Previous studies have demonstrated changes in DLG1 expression level and distribution during cell differentiation within squamous cervical and columnar intestinal epithelium (Watson et al., 2002; Cavatorta et al., 2004; Gardiol 


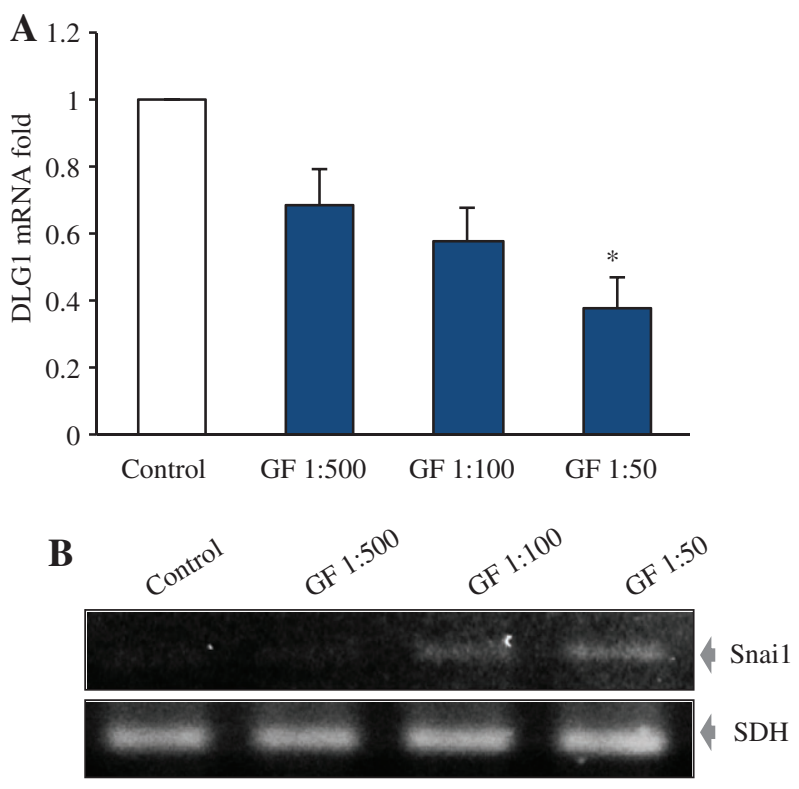

Figure 4: Growth factors negatively regulate DLG1 transcription. (A) Growth factors repress expression of DLG1 transcripts in a dose-dependent manner. Relative expression of DLG1 mRNA, after treatment of epithelial cells with the indicated dilutions of CGF commercial supplement (GF). The transcripts were determined by RT-qPCR, using SDH as internal reference gene. Results were shown as mean \pm SE and represent data from at least four independent experiments. ${ }^{\star} p<0.05$. (B) The transcriptional expression of Snai1 was increased by CGF treatment in a dose-dependent manner. Snai1 transcription was ascertained by semi-quantitative RT-PCR, using RNA from epithelial untreated cells (control) or after addition of the indicated amount of growth factors (GF). The levels of SDH were analysed as a control of equivalent amounts of cDNA.

et al., 2006). However, the pattern of DLG1 expression during differentiation was different in the two tissues. While, in squamous epithelium, DLG1 protein levels were reduced in cells of the most differentiated strata (Watson et al., 2002; Cavatorta et al., 2004), higher expression of DLG1 was observed in intestinal villous cells, in comparison with less differentiated cells of the crypt (Gardiol et al., 2006). Nevertheless, the expression of DLG1 during differentiation might be tissue-specific and might depend on the diverse signal transduction pathways involved in each biological context. Furthermore, little is known about the expression of this protein in the process of differentiation in other cell types.

In order to assess the importance of DLG1 expression during differentiation using another cell model, we chose the system of monocyte-to-macrophage differentiation. The PMA-stimulated THP-1 cell differentiation has been broadly used as a well-established system for the analysis of differentially expressed proteins (Tsuchiya et al., 1982; Poitz et al., 2013). In addition, and as shown in Figure 1,
THP-1 monocyte cells expressed DLG1 mRNA and, even more, these cells present alternative splicing at the $5^{\prime}$-UTR.

THP- 1 cells were treated with PMA (10 $\mathrm{ng} / \mathrm{ml})$ for $24 \mathrm{~h}$; within this time period the cells adhered to the cell culture plate and changed their morphology into a macrophage phenotype (Tsuchiya et al., 1982). Protein and RNA were extracted for evaluation of DLG1 expression from cells harvested before and after incubation with PMA. As can be seen in Figure 5A and B, DLG1 protein and total transcription levels decreased greatly following PMA induction. In differentiated cells, it was also apparent that the DLG1 protein exhibited retarded mobility in SDS-PAGE, which probably reflects post-translational modification such as phosphorylation or different spliced isoforms (Figure 5A). However, even though both short and large $5^{\prime}$-UTR transcript levels also diminished with the PMA treatment, the reduction was more pronounced for the short variant than for the large one (Figure 5C). Hence, relevant changes in the $5^{\prime}$-UTR short/large ratio were obtained (Figure 5C). Taken together, these data indicate that during monocyte differentiation, DLG1 expression is reduced and this may be regulated by transcriptional mechanisms with the participation of the alternative splicing in the DLG1 5'-UTR controlling the translational efficiency.

\section{Discussion}

In this study we report new insights into transcriptional and post-transcriptional mechanisms that may contribute to control DLG1 abundance. We first wanted to address the question whether the identified alternative short and large 5'-UTR isoforms (Cavatorta et al., 2011) were broadly expressed in different cell types. We included some cells where the importance of DLG1 expression had previously been established and where no data about the possible mechanism of expression regulation were available. DLG1 was shown to be a key regulator of lymphocyte proliferation and, together with other PDZ domain proteins, controls lymphocyte antigen response (Yoshida et al., 2008). In the nervous tissues, DLG1 functions as a synaptic scaffold that interacts with glutamate receptors and it was suggested a possible association of DLG1 with neurological disorders such as schizophrenia and autism (Facciuto et al., 2012). DLG1 also participates in the control of the proliferation of hepatocytes whose functions highly depends on polarisation (Duffy et al., 2007). We demonstrated that the alternative splicing in the $5^{\prime}$-UTR was a common and conserved event in all the cells analysed, with possible implications in the control of DLG1 abundance. However, these analyses were performed using cancer cells and 
A

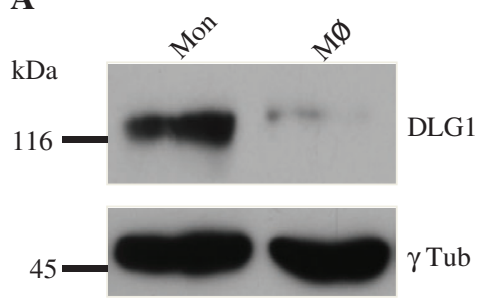

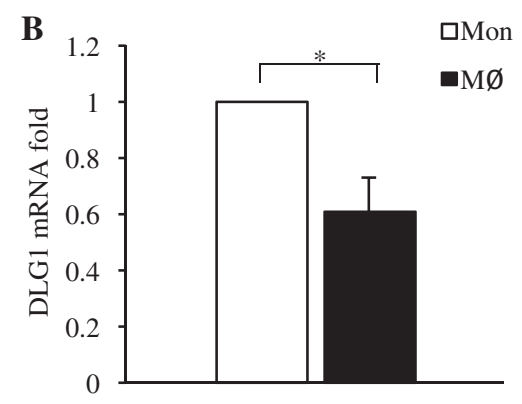

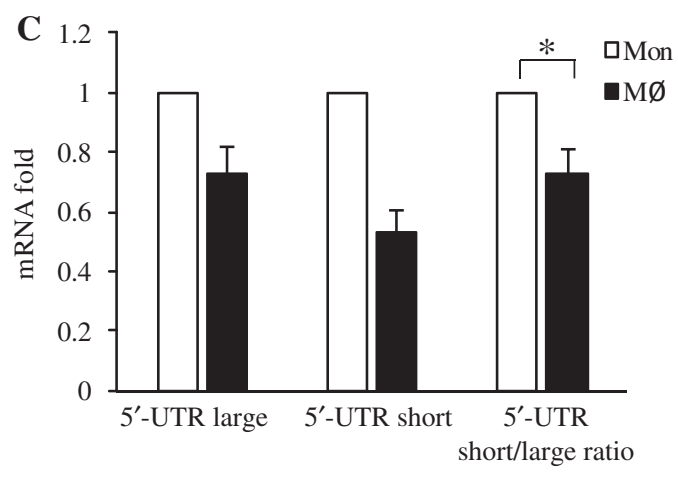

Figure 5: PMA-induced differentiation of monocytes to macrophages results in reduction of DLG1 expression.

(A) Protein levels were ascertained by Western blotting analysis, using THP-1 cells (monocytes, Mon) and THP-1 cells after the treatment with PMA (MØ), as described in Materials and methods. Antibodies used: anti-DLG1 (upper panel) or anti- $\gamma$ tubulin antibodies (as loading control, lower panel). (B) The DLG1 transcription is reduced after monocyte differentiation. Changes in total DLG1 mRNA level were quantified by RT-qPCR as described in Materials and methods. DLG1 mRNA contents were normalised to the SDH mRNA for all samples and the relative level of DLG1 mRNA in Mon was arbitrarily considered to be $1 .{ }^{*} p<0.05$. (C) The alternative splicing in the DLG1 $5^{\prime}$-UTR is reduced during monocyte differentiation. Variation of each $5^{\prime}$-UTR mRNA isoforms level for M $\emptyset$ relative to Mon (data arbitrarily considered to be 1) is shown. For quantification of each alternative DLG1 $5^{\prime}$-UTR we performed RT-qPCR as described under Materials and methods. DLG1 $5^{\prime}$-UTR mRNA contents were normalised to the SDH mRNA contents for all samples. Relative short/large $5^{\prime}$-UTR mRNA fold is also shown for monocytes and macrophages. Short/large DLG1 $5^{\prime}$-UTR mRNA from monocyte cells was arbitrarily considered to be $1 .{ }^{*} p<0.05$. In all cases, results were shown as mean $\pm \mathrm{SE}$ and represent data from three independent experiments.

additional studies are necessary to understand the real impact on DLG1 protein levels in each specific cell type.

In epithelial cells, cell-cell adhesion induces the expression of DLG1 and its recruitment to the lateral plasma membrane at cell contacts (Reuver and Garner, 1998). The finding that cells growing with fewer membrane junctions exhibit low levels of DLG1 expression suggested that cellular mechanism may be regulating DLG1 levels in response to the degree of cell confluence (Mantovani et al., 2001). This hypothesis proves interesting when considering that cells from a variety of tumours lose the ability to form cell junctions, as well as considering the loss of DLG1 expression in undifferentiated cancers (Facciuto et al., 2012). We have shown a major increase in DLG1 levels in high cell density conditions and this could be explained by activation at the transcriptional level, together with an enhancement in the 5'-UTR alternative splicing that renders a higher proportion of the short isoform RNA, with an upper efficiency of translation.
These findings highlight the fact that DLG1 is subjected to a complex pattern of dynamic regulation when cells reach confluence, hinting that this protein plays an active role in the organisation of epithelial junctions which hold the cells together and that, in turn, DLG1 may negatively regulate cell cycle progression upon cell contact (Ishidate et al., 2000; Stephenson et al., 2007). In fact, DLG1 is able to interact through the PDZ domains with both APC and PTEN tumour suppressors with important consequences for cell cycle arrest, inhibition of $S$ phase entry and reduction of proliferating signalling (Sotelo et al., 2012). In this report we demonstrated that in agreement with this, DLG1 is down-regulated when the $S$ phase is stimulated and this may account for a decrease in DLG1 RNA level as shown in Figure 3. However, the relation between both $5^{\prime}$-UTR remains unchanged during the $S$ entry, suggesting that the down-regulation of DLG1 protein in this process is not regulated at the translational level. It is possible to speculate that the progression into the cell cycle promotes the 
synthesis or repression of different transcription factors that can differentially regulate the transcription of DLG1. In this sense the Snail family of transcription regulators represses the DLG1 promoter activity by specifically binding to the promoter (Cavatorta et al., 2008). Snai1 also behaves as an epidermal growth factor effector and probably this event may be responsible for the down-regulation of DLG1 in response to growth factors, as it was shown in Figure 4 (De Craene et al., 2005; Cavatorta et al., 2008). However, it is not possible to discard that other not yet identified mitogen-induced transcription factors can regulate DLG1 levels during cell cycle progression.

In previous studies we showed that, during epithelial cell differentiation, in the environment of a squamous epithelium there is a clear reduction in DLG1 levels as cells move and differentiate towards the upper most stratus (Cavatorta et al., 2004). We wanted to extend our investigations in order to analyse if this also holds true in other cell type models. We studied the changes in DLG1 abundance for monocyte-to-macrophage transition performing the well-established differentiation model using PMA treatment. Monocytes are blood circulating cells and, after adhesion to the vessel wall, migrate into tissues and differentiate into macrophages, which is an important process under physiological and pathological conditions. It was shown that other PDZ polarity proteins, such as partitioning defective 3 (PAR 3), are associated with monocytes polarised cell migration, indicating the importance of cell polarity proteins in the biological functions of monocytes (Tamehiro et al., 2009). We observed that DLG1 protein levels decrease during monocyte-to-macrophage differentiation, together with a reduction in the transcription level and in the $5^{\prime}$-UTR alternative splicing rate, resulting in a relatively higher proportion of the large RNA isoform. PMA-induced differentiation correlates with increased nuclear translocation of NFkB, interestingly, we identified binding sites for NFkB in the DLG1 promoter region (Cavatorta et al., 2008), and it is possible to speculate that this contributes to the shift in DLG1 transcriptional level. After migration into the tissues and differentiation into macrophages, the cells found a hypoxic milieu; moreover, it was observed an increased expression of the hypoxiainducible factors (HIFs) after PMA stimulation (Poitz et al., 2013). HIFs have been shown to regulate the transcription of adhesion proteins and probably, this mechanism may also be implicated in the down-regulation of DLG1 during the monocytes differentiation (Cheng et al., 2013).

In summary, in this report we got insight into changes in DLG1 levels in different biological environments. However, more work remains to be done and future studies will need to address the specific molecular mechanisms that regulate the $5^{\prime}$-UTR alternative splicing and the different signalling pathways and downstream cross talks involved in DLG1 transcriptional regulation.

\section{Materials and methods}

\section{Cell culture and antibodies}

HEK293, HaCaT and Caco-2 epithelial cells were grown in Dulbecco's modified Eagle's medium (DMEM) (Gibco, Grand Island, NY, USA). HepG2 cells (human hepatocellular carcinoma) and SH-SY5Y cells (human neuroblastoma) were grown in DMEM:Ham's F12 medium. Jurkat cells (human T cell leukaemia), K562 cells (human myelogenous leukaemia) and THP-1 cells (human monocyte-derived leukaemia) were grown in RPMI1640 (Gibco, Grand Island, NY, USA) medium. All media were supplemented with $10 \%$ (v/v) foetal bovine serum (FBS) (PAA Laboratories GmbH, Paschin, Austria).

Antibodies used were: mouse monoclonal anti-DLG1 (2D11), mouse monoclonal anti-cyclinE (HE12) (Santa Cruz Biotechnology, Dallas, TX, USA), and mouse monoclonal anti- $\gamma$ tubulin (T6557) (Sigma Aldrich, Saint Louis, MO, USA).

For the analysis of cell growth factors treatment over DLG1 expression, cells were cultured in DMEM (Gibco, Grand Island, NY, USA) supplemented with 5\% FBS (PAA Laboratories GmbH, Pasching, Austria) and either incubated or not for $24 \mathrm{~h}$ previous to RNA extraction, with different dilutions of the Defined Keratinocyte-SFM (Gibco, Grand Island, NY, USA) commercial supplement containing insulin, and epidermal and fibroblast grown factor (CGF).

\section{RNA isolation, cDNA synthesis, RT-PCR and RT-qPCR}

Total RNA was purified using Trizol according to the manufacturer's protocol (Invitrogen, Carlsbad, CA, USA). Synthesis of cDNA was obtained from $2 \mu \mathrm{g}$ of RNA using $200 \mathrm{U}$ MMuLV RevertAid reverse transcriptase (Fermentas, Thermo Fisher Scientific, Glen Burnie, MD, USA) and oligo(dT) primers. For RT-PCR, each alternative DLG1 5'UTR was specifically amplified using different sense primers as previously described (Cavatorta et al., 2011) (F3, for DLG1 5'-UTR large 5'-TGT CTC GGT ATG TGC GCC TT-3'; F4, for DLG1 5'-UTR short, 5'-TGT CTC GGT GTG TGC CCT CTT-3') and a common antisense primer (R, 5'-AGC TGT CTG TCT TCA GTT TGG CT-3'). Human Snai1 transcript was amplified using oligonucleotides: RT-hSnai1For (5'-GCT GCA GGA CTC TAA TCC AGA GT-3') and RT-hSnai1Rev (5'-AGC CTT TCC CAC TGT CCT CAT CT-3'). RT-PCR was performed using $2 \mu \mathrm{l}$ of template cDNA, $2.5 \mathrm{mM} \mathrm{MgCl}_{2}, 0.4 \mathrm{~mm}$ dNTPs and $20 \mathrm{pmol}$ of each primer for 27 cycles of $94^{\circ} \mathrm{C}$ for $40 \mathrm{~s}, 56^{\circ} \mathrm{C}$ for $40 \mathrm{~s}$, and $72^{\circ} \mathrm{C}$ for $40 \mathrm{~s}$. RT-qPCR analysis was performed using Eva Green qPCR Mezcla Real (Biodynamics, Buenos Aires, Argentina). For these analyses, the primers used were the same as described above except for DLG1 5'-UTR large transcript where a new sense primer was designed with the following sequence: F-large, 5'-GGG CTA GGG CAA GGT GTG T-3'. Furthermore, total DLG1 cDNA was amplified using primers that target the coding region [DLG-F, 5'-CAA GCA GCC TTA GCC CTA GTG TA-3' (sense), and DLG-R, 5'-CATGAACCAATTCTGGACCTATCA-3' (antisense)]. For normalisation, the reference gene $S D H$ was quantified using SDH-F $5^{\prime}$-GCA CAC CCT GTC CTT TGT-3' (sense) and SDH-R 5'-CAC AGT CAG CCT CGT TCA-3' 
(antisense) oligonucleotides. The RT-qPCR reaction conditions were set at $95^{\circ} \mathrm{C}$ for $5 \mathrm{~min}$ followed by 40 cycles of denaturation $\left(95^{\circ} \mathrm{C}\right.$ for $15 \mathrm{~s})$, annealing $\left(60^{\circ} \mathrm{C}\right.$ for $\left.15 \mathrm{~s}\right)$ and extension $\left(72^{\circ} \mathrm{C}\right.$ for $\left.20 \mathrm{~s}\right)$, with a single acquisition of fluorescence levels at the end of each extension step. Dissociation curve analysis of amplification products was performed at the end of each PCR reaction to confirm that only one PCR product was amplified and detected.

Data were analysed using the threshold cycle $(\mathrm{Ct})$ relative quantification method ( $\Delta \Delta \mathrm{Ct}$, Livak and Schmittgen, 2001). Briefly, gene expression levels were normalised by the SDH reference gene. The relative abundance of the transcripts was calculated from $2^{(-\Delta \mathrm{Ct})}$, where $\Delta \mathrm{Ct}=\mathrm{Ct}$ DLG1, short or large $5^{\prime}$-UTR $-\mathrm{Ct} \mathrm{SDH}$. Results are considered as differences in gene expression during the different biological processes and are expressed in relation to a given condition for each process. They were calculated using the $2^{(-\Delta \Delta \mathrm{Ct})}$ method.

\section{Analysis of cell cycle profiles by fluorescence-activated cell sorting (FACS)}

CaCo-2 cells were seeded at a density of $3 \times 10^{4}$ cells in $60 \mathrm{~mm}$ dishes and cultured in DMEM medium containing 0.02\% FBS for $48 \mathrm{~h}$ and then switched to DMEM containing 10\% FBS medium and cultured for $24 \mathrm{~h}$. Cells were harvested at different times after serum repletion and DNA content was assessed by propidium iodide (PI) staining and FACS analysis as previously described (Darzynkiewicz et al., 2001). Briefly, cells were fixed in $8 \mathrm{ml}$ of cold $70 \%$ ethanol in PBS, washed in PBS and stained with PI (PI, $50 \mu \mathrm{g} / \mathrm{ml}$ of final concentration). Flow cytometry assay was performed as previously described (Darzynkiewicz et al., 2001) using a FACS Aria II cell sorter (Becton-Dickinson, San Jose, CA, USA) equipped with a 488-nm argon laser. Subsequent data analysis was made employing WindMDI software (Joseph Trotter, Scripps Research Institute, La Jolla, CA, USA). For each sample, the forward versus right-angle scatter cytogram was used to exclude debris and aggregates. A minimum of $10^{4}$ cells were analysed with linear amplification for PI fluorescence that was collected with a $600-\mathrm{nm}$ long pass filter. Cell distribution in the different phases of the cell cycle was estimated using CychleRed Software (Terry Hoy, Cardiff University, Cardiff, UK).

\section{Western blotting}

Western blot analyses were carried out as previously described (Gardiol et al., 1999). Briefly, cells were harvested in E1A extraction buffer (250 mm NaCl, 0.1\% NP40, 50 mm HEPES pH 7.0, 1 mm $\mathrm{MgCl}_{2}$ ) containing Halt Protease Inhibitor single use cocktail (Thermo Scientific Pierce, Rockford, USA). Equal amounts of proteins were separated by SDS-PAGE and transferred to nitrocellulose. Specific protein levels were determined by immunoblot analysis using the appropriate primary antibodies as indicated in the text. Blots were developed using the SuperSignal West Pico Chemiluminescent Substrate reagent (Thermo Scientific Pierce, Rockford, IL, USA).

\section{Phorbol myristate acetate (PMA)-induced differentiation of THP-1 cells}

THP-1 monocytic cells $\left(1 \times 10^{6}\right)$ were differentiated into macrophages in $60 \mathrm{~mm}$ dishes containing $3.5 \mathrm{ml}$ of the RPMI medium in the presence of $10 \mathrm{ng} / \mathrm{ml}$ of PMA (Sigma, St Louis, MO, USA) over $48 \mathrm{~h}$. After incubation, nonattached cells were removed by aspiration, and the adherent cells (differentiated monocytes) were washed twice with PBS and harvested for RNA and protein analysis.

\section{Statistical analysis}

RT-qPCR data are shown with standard error (SE) of the mean as the mean \pm SE and are representative for at least three independent experiments. In general, statistical significances were calculated using an unpaired two-tailed Student's $t$-test. The statistical significance of the data shown in Figure $3 \mathrm{~F}$ was obtained by the Scheffé test. $p$ Values $<0.05$ were considered to be significant.

Acknowledgments: We gratefully acknowledge Dolores Campos for her excellent technical support and help with the cell culture. We also thank María Robson, Mariana de Sanctis, Geraldine Raimundo, Romina Ricardo and Carolina Perret from the English Department (Facultad de Ciencias Bioquímicas y Farmacéuticas, UNR) for the English language editing of the manuscript. This work was partially supported by a research grant from the Agencia de Promoción Científica y Tecnológica (Argentina, PICT 2008-0421) and from the Province of Santa Fe, Argentina.

\section{References}

Assemat, E., Bazellieres, E., Pallesi-Pocachard, E., Le Bivic, A., and Massey-Harroche, D. (2008). Polarity complex proteins. Biochim. Biophys. Acta 1778, 614-630.

Bilder, D. (2004). Epithelial polarity and proliferation control: links from the Drosophila neoplastic tumor suppressors. Genes Dev. 18, 1909-1925.

Boussioutas, A., Li, H., Liu, J., Waring, P., Lade, S., Holloway, A., Taupin. D., Gorringe, K., Haviv, I., Desmond, P., et al. (2003). Distinctive patterns of gene expression in premalignant gastric mucosa and gastric cancer. Cancer Res. 63, 2569-2577.

Cavatorta, A.L., Fumero, G., Chouhy, D., Aguirre. R., Nocito, A. L., Giri, A., Banks, L., and Gardiol, D. (2004). Differential expression of the human homologue of drosophila discs large oncosuppressor in histologic samples from human papillomavirus-associated lesions as a marker for progression to malignancy. Int. J. Cancer 111, 373-380.

Cavatorta, A.L., Giri, A., Banks, L., and Gardiol, D. (2008). Cloning and functional analysis of the promoter region of the human Disc large gene. Gene 424, 87-95.

Cavatorta, A.L., Facciuto, F., Valdano, M., Marziali, F., Giri, A., Banks, L., and Gardiol, D. (2011). Regulation of translational efficiency by different splice variants of the Disc Large 1 oncosuppressor 5'-UTR. FEBS J. 278, 2596-2608.

Cheng, J., Klausen, C., and Leung, P. (2013). Hypoxia-inducible factor $1 \alpha$ mediates epidermal growth factor-induced downregulation of E-cadherin expression and cell invasion in human ovarian cancer cells. Cancer Lett. 329, 197-220. 
Darzynkiewicz, Z., Juan, G., and Bedner, E. (2001). Determining cell cycle stages by flow cytometry. In: Current Protocols in Cell Biologym, J.S. Bonifacino, M. Dasso, J. Harford, J. Lippincott-Schwartz and K. Yamada, eds. (Hoboken, NJ, USA: John Wiley Publisher). Chapter 8, Unit 84.

De Craene, B., van Roy, F., and Berx, G. (2005). Unraveling signalling cascades for the Snail family of transcription factors. Cell Signal. 17, 535-547.

Dikstein, R. (2012). Transcription and translation in a package deal: the TISU paradigm. Gene 491, 1-4.

Duffy, H., lacobas, I., Hotchkiss, K., Hirst-Jensen, B., Bosco, A., Dandachi, N., Dermietzel, R., Sorgen, P. and Spray, D.C. (2007). The gap junction protein connexin 32 interacts with the $\mathrm{Src}$ homology $3 /$ hook domain of discs large homolog 1 . J. Biol. Chem. 282, 9789-9796.

Facciuto, F., Cavatorta, A., Valdano, M., Marziali, F., and Gardiol, D. (2012). Differential expression of PDZ domain-containing proteins in human diseases-challenging topics and novel issues. FEBS J. 279, 3538-3548.

Frese, K., Latorre, I., Chung, S., Caruana, G., Bernstein, A., Jones, S., Donehower, L., Justice, M., Garner, C., and Javier, R. (2006). Oncogenic function for the Dlg1 mammalian homolog of the Drosophila discs-large tumor suppressor. EMBO J. 25, 1406-1417.

Fuja, T., Lin, F., Osann, K., and Bryant, P. (2004). Somatic mutations and altered expression of the candidate tumor suppressors CSNK1 epsilon, DLG1, and EDD/hHYD in mammary ductal carcinoma. Cancer Res. 64, 942-951.

Gardiol, D., Kuhne, C., Glaunsinger, B., Lee, S., Javier, R., and Banks, L. (1999). Oncogenic human papillomavirus E6 proteins target the discs large tumour suppressor for proteasomemediated degradation. Oncogene 18, 5487-5496.

Gardiol, D., Zacchi, A., Petrera, F., Stanta, G., and Banks, L. (2006). Human discs large and scrib are localized at the same regions in colon mucosa and changes in their expression patterns are correlated with loss of tissue architecture during malignant progression. Int. J. Cancer 119, 1285-1290.

Ishidate, T., Matsumine, A., Toyoshima, K., and Akiyama, T. (2000). The APC-hDLG complex negatively regulates cell cycle progression from the G0/G1 to S phase. Oncogene 19, 365-372.

Laprise, P., Viel, A., and Rivard, N. (2004). Human homolog of disc-large is required for adherens junction assembly and differentiation of human intestinal epithelial cells. J. Biol. Chem. 279, 10157-10166.

Livak, K. and Schmittgen, T. (2001). Analysis of relative gene expression data using real-time quantitative PCR and the $2^{-\Delta \Delta C T}$ method. Methods 25, 402-408.

Lue, R., Marfatia, S., Branton, D., and Chishti, A. (1994). Cloning and characterization of hdlg: the human homologue of the Drosophila discs large tumor suppressor binds to protein 4.1. Proc. Natl. Acad. Sci. USA 91, 9818-9822.

Mantovani, F., Massimi, P., and Banks, L. (2001). Proteasomemediated regulation of the hDlg tumour suppressor protein. J. Cell Sci. 114, 4285-4292.

McLaughlin, M., Hale, R., Ellston, D., Gaudet, S., Lue, R., and Viel, A. (2002). The distribution and function of alternatively spliced insertions in hDlg. J. Biol. Chem. 277, 6406-6412.

Poitz, D., Augstein, A., Gradehand, C., Ende, G., Schmeisser, A., and Strasser, R. (2013). Regulation of the Hif-system by micro-RNA
17 and 20a-role during monocyte-to-macrophage differentiation. Mol. Immunol. 56, 442-451.

Reuver, S. and Garner, C. (1998). E-cadherin mediated cell adhesion recruits SAP97 into the cortical cytoskeleton. J. Cell Sci. 111, 1071-1080.

Roberts, S., Delury, C., and Marsh, E. (2012). The PDZ protein human discs large: the "Jekyll and Hyde" of the epithelial polarity proteins. FEBS J. 279, 3949-3958.

Sotelo, N., Valiente, M., Gil, A., and Pulido, R. (2012). A functional network of the tumor suppressors APC, hDlg, and PTEN, that relies on recognition of specific PDZ-domains. J. Cell. Biochem. 113, 2661-2670.

Stephenson, L., Sammut, B., Graham, D., Chan-Wang, J., Brim, K., Huett, A., Miletic, A., Kloeppel, T., Landry, A., Xavier, R., et al. (2007). DLGH1 is a negative regulator of T-lymphocyte proliferation. Mol. Cell. Biol. 27, 7574-7581.

Subbaiah, V., Narayan, N., Massimi, P., and Banks, L. (2012). Regulation of the DLG tumor suppressor by $\beta$-catenin. Int. J. Cancer 131, 2223-2233.

Szymanowska-Narloch, A., Jassem, E., Skrzypski, M., Muley, T., Meister, M., Dienemann, H., Taron, M., Rosell, R., Rzepko, R., Jarzab, M., et al. (2013). Molecular profiles of non-small cell lung cancers in cigarette smoking and never-smoking patients. Adv. Med. Sci. 58, 196-206.

Tamehiro, N., Mujawar, Z., Zhou, S., Zhuang, D.Z., Hornemann, T., von Eckardstein, A., and Fitzgerald, M. (2009). Cell polarity factor Par3 binds SPTLC1 and modulates monocyte serine palmitoyltransferase activity and chemotaxis. J. Biol. Chem. 284, 24881-24890.

Tsuchiya, S., Kobayashi, Y., Goto, Y., Okumura, H., Nakae, S., Konno, T., and Tada, K. (1982). Induction of maturation in cultured human monocytic leukemia cells by a phorbol diester. Cancer Res. 42, 1530-1536.

Valiente, M., Andres-Pons, A., Gomar, B., Torres, J., Gil, A., Tapparel, C., Antonarakis, S., and Pulido, R. (2005). Binding of PTEN to specific PDZ domains contributes to PTEN protein stability and phosphorylation by microtubule-associated serine/threonine kinases. J. Biol. Chem. 280, 28936-28943.

Vieira, V., de la Houssaye, G., Lacassagne, E., Dufier, J., Jais, J., Beermann, F., Menasche, M., and Abitbol, M. (2008). Differential regulation of Dlg1, Scrib, and Lgl1 expression in a transgenic mouse model of ocular cancer. Mol. Vis. 14, 2390-2403.

Watson, R., Rollason, T., Reynolds, G., Murray, P., Banks, L., and Roberts, S. (2002). Changes in expression of the human homologue of the Drosophila discs large tumour suppressor protein in high-grade premalignant cervical neoplasias. Carcinogenesis 23, 1791-1796.

Yoshida, S., Higuchi, M., Shoji, T., Yoshita, M., Ishioka, K., Takahashi, M., Oie, M., Tanaka, Y., Uchiyama, M., and Fujii, M. (2008). Knockdown of synapse-associated protein Dlg1 reduces syncytium formation induced by human T-cell leukemia virus type 1. Virus Genes 37, 9-15.

Zimmermann, T., Kashyap, A., Hartmann, U., Otto, G., Galle, P., Strand, S., and Strand, D. (2008). Cloning and characterization of the promoter of Hugl-2, the human homologue of Drosophila lethal giant larvae (lgl) polarity gene. Biochem. Biophys. Res. Commun. 366, 1067-1073. 